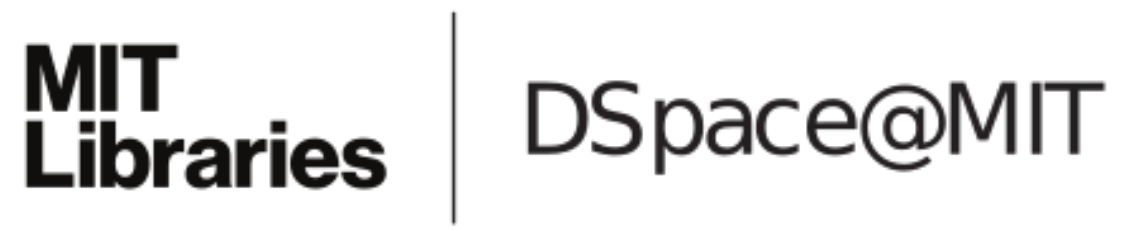

\author{
MIT Open Access Articles
}

Enhanced cycle slip detection method for dualfrequency BeiDou GEO carrier phase observations

The MIT Faculty has made this article openly available. Please share how this access benefits you. Your story matters.

Citation: Ju, Bing, Defeng Gu, Xiao Chang, Thomas A. Herring, Xiaojun Duan, and Zhengming Wang. "Enhanced Cycle Slip Detection Method for Dual-Frequency BeiDou GEO Carrier Phase Observations." GPS Solutions 21, 3 (February 14, 2017): 1227-1238.

As Published: https://doi.org/10.1007/s10291-017-0607-8

Publisher: Springer-Verlag

Persistent URL: https://hdl.handle.net/1721.1/121403

Version: Author's final manuscript: final author's manuscript post peer review, without publisher's formatting or copy editing

Terms of use: Creative Commons Attribution 4.0 International license 


\title{
Enhanced cycle slip detection method for dual-frequency BeiDou GEO carrier phase observations
}

\author{
Bing $\mathrm{Ju}^{1}$ (D) Defeng $\mathrm{Gu}^{1} \cdot$ Xiao Chang ${ }^{1} \cdot$ Thomas A. Herring $^{2} \cdot$ Xiaojun Duan ${ }^{1} \cdot$ \\ Zhengming Wang ${ }^{1}$
}

Received: 12 November 2016/Accepted: 6 February 2017

(C) Springer-Verlag Berlin Heidelberg 2017

\begin{abstract}
Cycle slip detection is one of the essential steps for high-precision GNSS data processing when carrier phase observations are involved, such as in precise point positioning (PPP) and precise orbit determination (POD). A number of algorithms have been developed since the 1980s and are effective for processing dual-frequency GPS. However, the emerging BeiDou navigation satellite system in China brings some new challenges for these existing algorithms, especially when small cycle slips occur more frequently. In this study, a large number of 1-cycle slips have been found in low-elevation BeiDou GEO carrier phase observations, which are collected by receivers of the IGS multi-GNSS experiment. Such small cycle slips should be identified and repaired, if possible, before PPP and POD processing. We propose an enhanced cycle slip detection method based on the series of dual-frequency phase geometry-free combinations. A robust polynomial fit algorithm and a general autoregressive conditional heteroskedastic modeling technique is employed to provide an adaptive detection threshold, which allows identification of such small cycle slips with high reliability. Simulated and real data tests reveal that the proposed method has both high sensitivity and low false-alarm rate even in the case of ionospheric scintillation.
\end{abstract}

\footnotetext{
Bing Ju

supice@nudt.edu.cn

$\triangle$ Defeng Gu

gudefeng@nudt.edu.cn

1 College of Science, National University of Defense Technology, Changsha 410073, China

2 Department of Earth, Atmospheric and Planetary Science, Massachusetts Institute of Technology, Cambridge, MA 02139, USA
}

Keywords BDS - GEO - Cycle slip detection - Ionospheric scintillation $\cdot$ GARCH

\section{Introduction}

Carrier phase observations are always used in precise GNSS applications, such as precise point positioning (PPP) and precise orbit determination (POD). While carrier phase observations are more accurate than pseudoranges, they may suffer unpredictable but frequent cycle slips in a continuous tracking arc. A cycle slip presents a sudden jump of phase ambiguity by an integer number of cycles, which could be caused by interruption of the GNSS transmitted signal, low signal-to-noise ratio, and intensive ionospheric scintillation. Whatever the reason for the slips, such undesirable discontinuities should be identified before the carrier phases are used as high-precision measurements.

Cycle slip detection using dual-frequency GNSS observations has been investigated for many years, and various algorithms have been proposed since the 1980s. Examples are phase ionospheric residual method (Goad 1985), the TurboEdit algorithm (Blewitt 1990), high-order time difference method (Kleusberg et al. 1993), and Bayesian approach (de Lacy et al. 2008). A reliable cycle slip detection method with both high sensitivity and low false-alarm rate is always desirable, but unfortunately, it is hard to realize in practice. The TurboEdit algorithm, which employs the Hatch-Melbourne-Wübbena (HMW) combination (Hatch 1982) as well as the ionospheric/geometry-free combinations, is most widely used for cycle slip detection on a single receiver. While the HMW combination is both geometryfree and ionospheric-free, the sensitivity of such combination is limited by large pseudorange noise. Therefore, it is hard for the TurboEdit algorithm to detect cycle slips as 
small as 1-2 cycles in some noisy situations (Cai et al. 2013). In addition, the HMW combination is insensitive to cycle slips that are the same on both frequencies. To enhance the detectability for small cycle slips, the phase geometry-free combination, i.e., L1-L2, is often preferred since it only depends on carrier phase observations. However, the performance of such combination is subject to ionospheric disturbances (Banville and Langley 2013). If the ionospheric variations are smooth, the ionospheric delay term in the L1L2 combination can be largely mitigated by a time-differenced or polynomial fitting algorithm. Under this assumption, even a 1-cycle slip occurring on L1 or L2, or simultaneously on both frequencies, can be detected (de Lacy et al. 2008). When the ionospheric delay shows rapid variations, most of the L1-L2 based methods will lose the sensitivity to small cycle slips and result in either high falsealarm rate or missed detections. Given this, some further efforts are needed to account for the effect of ionospheric variations in cycle slip detection. Liu (2011) estimates the ionospheric total electron content rate (TECR), which is essentially calculated based on the L1-L2 combination, to capture the physical characteristics of ionospheric variations. If the calculated TECR is larger than a predefined threshold, then cycle slips are considered to exist at the current epoch. However, this approach is only effective for data with a high sampling rate, such as $1 \mathrm{~Hz}$. For commonly used 30-s sampling data, it is difficult to detect those small cycle slips due to the poor approximation of the TECR. To detect cycle slips during periods of ionospheric scintillation, Ji et al. (2013) employs a fourth-order difference of an ionospheric-free but not geometry-free testing quantity, which is formed by a single difference between phase ionospheric-free combinations of two satellites. Though the effect of ionosphere is completely removed, one cannot state which satellite is involved in cycle slip at the moment. In addition, the case of sequential cycle slips makes it difficult for this high-order difference method to determine the exact location of each individual cycle slip. Banville and Langley (2013) treat the dual-frequency cycle slip detection as a parameter estimation problem in a time-differenced model. The ionospheric delay is estimated in their model. The main drawback of this method is the dependence of external information, such as precise satellite orbits and clock biases. In summary, how to properly deal with the ionospheric delay is still a key issue to improve the ability of cycle slip detection using dual-frequency GNSS observations.

The emerging Chinese BeiDou navigation satellite system (BDS) brings some new challenges to current dualfrequency cycle slip detection methods. Most of the research on cycle slip detection is based on simulations; the observed features of cycle slip in BDS observations are seldom discussed. During our routine data processing for the Multi-GNSS Experiment (MGEX) network
(Montenbruck et al. 2014), extensive small cycle slips have been found in BDS carrier phase observations, in particular for the satellites in geostationary earth orbit (GEO). These cycle slips are often only 1 cycle and can happen on B1 or B2, or both B1 and B2 frequencies simultaneously. These characteristics greatly increase the difficulty of dual-frequency cycle slip detection. While triple frequencies are transmitted by all BDS satellites and some triple-frequency-based methods have been proposed, such as $\mathrm{Wu}$ et al. (2010), de Lacy et al. (2012), Huang et al. (2016) and Zhang and Li (2016), many GNSS receivers do not track triple frequencies at the present time (Chen et al. 2016). In addition, most of the current PPP and POD algorithms are still based on dual-frequency observations. There would be a potential risk of contamination if cycle slips occur frequently but fail to be repaired on the third frequency. Thus, the dual-frequency cycle slip detection method will still be important in practice until triple-frequency techniques are fully exploited.

The motivation of this study is to provide an enhanced dual-frequency cycle slip detection method, which is not only able to detect 1-cycle slips in BDS observations but also has a low false-alarm rate in the case of ionospheric scintillation. In addition, the high sampling rate should not be necessary for the proposed method. Based on the dualfrequency phase geometry-free combination, which is sensitive to small cycle slips, the nonstationary time series analysis method is applied to deal with the ionospheric variations. The HMW combination is mainly used as a complement to avoid missing detection for those cycle slip combinations that are insensitive to the phase geometryfree combination. On the other hand, the HMW combination will also be used to determine the size of individual cycle slip along with the phase geometry-free combination.

In the following, we first present the phenomenon of extensive 1-cycle slips in BDS carrier phase observations. Then, we propose a robust polynomial fit algorithm and a general autoregressive conditional heteroskedastic (GARCH) model for the nonstationary time series of phase geometry-free combinations. Finally, the performance of the proposed method is tested by both simulated and real data sets.

\section{Extensive 1-cycle slips in BeiDou GEO observations}

The Chinese navigation satellite system BeiDou, abbreviated as BDS and earlier referred to as COMPASS, has provided positioning, navigation and timing (PNT) services for Asia-Pacific region since December 27, 2012 (CSNO 2013). Distinguished from other global navigation satellite system (GNSS), such as GPS, GLONASS and Galileo, the 
BDS employs a mixed-type constellation utilizing both geostationary earth orbits (GEO) and inclined geostationary earth orbits (IGSO) aside from medium earth orbits (MEO) to ensure a good regional PNT performance (Montenbruck et al. 2013). The constellation of BDS presently consists of 13 active satellites, including five GEOs, five IGSOs, and three MEOs (Guo et al. 2016).

As a principal part of the current regional and future global BDS constellation, the GEOs are employed as navigation satellites for the first time and have proven to be effective for regional PNT enhancement (Montenbruck et al. 2013). The quality of carrier phase observations is crucial for orbit determination of GEO satellites and other relevant precise applications. However, extensive small cycle slips have been found in some BeiDou GEO carrier phase observations. This phenomenon can often be observed when a GEO satellite is visible at a ground station but with low tracking elevation, i.e., below $30^{\circ}$. As shown in Table 1, such cycle slips are seen in six selected MGEX stations. According to a preliminary analysis, these cycle slips have nothing to do with receiver type or satellite PRN.
So it is a common phenomenon rather than a single receiver or satellite induced problem. In addition, due to the nearly invariable geometry between a GEO satellite and a static receiver, this phenomenon can be observed almost every day.

Figure 1 illustrates the cycle slips in BDS C05 carrier phase observations, which are collected at station JFNG with 30-s sampling rate over a period of $24 \mathrm{~h}$. The significant discontinuities can be observed from the series of L1-L2. The first-order difference of L1-L2 reveals that most of these cycle slips occurring on B1 and B2 frequencies are $( \pm 1,0),(0, \pm 1),(1,1)$ or $(-1,-1)$. Although the HMW combination can identify some apparent jumps, it is hard to detect all such small and frequent cycle slips due to the disturbances of large code errors. In addition, the HMW combination is insensitive to identical cycle slip on B1 and B2 frequencies, such as $(1,1)$ and $(-1,-1)$. Furthermore, these cycle slips can also be partly confirmed by a triple-frequency phase geometry-free and ionosphericfree combination $L_{\mathrm{GIF}}$ (Montenbruck et al. 2013). However, it should be mentioned that not all of the cycle slips
Table 1 Cycle slips in dualfrequency (B1 and B2) BeiDou GEO observations collected by 6 MGEX stations with $30 \mathrm{~s}$ sampling rate over a period of $24 \mathrm{~h}$ on September 1, 2015
Fig. 1 Cycle slips in BDS C05 carrier phase observations collected by station JFNG on September 1, 2015. The horizontal dashed lines in the lower left panel indicate values of $\pm \lambda_{2}, \pm \lambda_{1}$ and $\pm\left(\lambda_{1}-\lambda_{2}\right)$, respectively. The colored triangles indicate cycle slips

\begin{tabular}{llcrccc}
\hline Station & Receiver type & Latitude $\left({ }^{\circ}\right)$ & Longitude $\left(^{\circ}\right)$ & PRN & Elevation $\left(^{\circ}\right)$ & \# of Cycle slip \\
\hline JFNG & TRIMBLE NETR9 & 30.516 & 114.491 & C05 & $20.0-21.5$ & 108 \\
REUN & SEPT POLARX4 & -21.208 & 55.572 & C03 & $23.9-25.1$ & 32 \\
WROC & LEICA GR25 & 51.113 & 17.062 & C02 & $7.6-8.3$ & 85 \\
DYNG & TRIMBLE NETR9 & 38.078 & 23.932 & C02 & $17.5-18.1$ & 209 \\
THTG & LEICA GR10 & -17.577 & -149.606 & C01 & $9.5-10.7$ & 257 \\
CAS1 & TRIMBLE NETR9 & -66.283 & 110.519 & C04 & $5.5-7.5$ & 13 \\
\hline
\end{tabular}
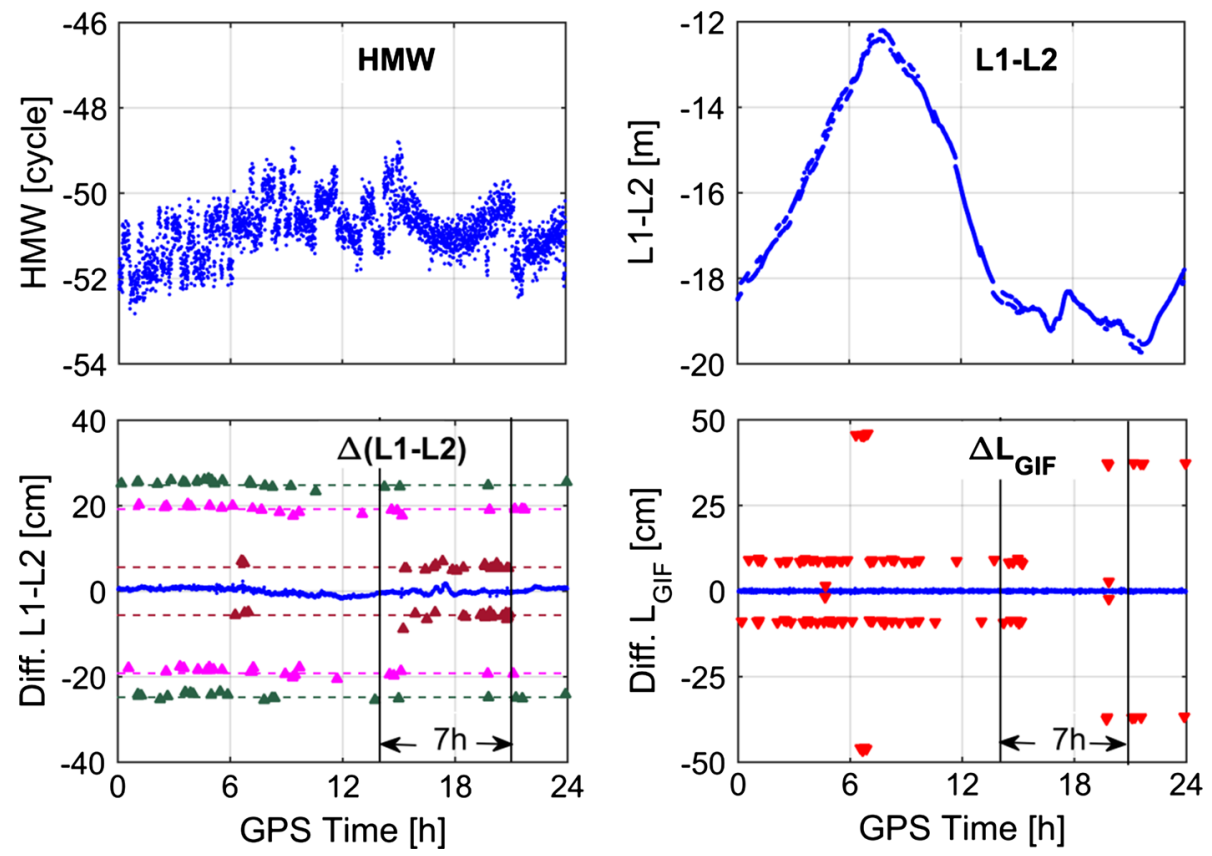
Fig. 2 Cycle slips in BDS C04 carrier phase observations collected by station CAS1 on September 1, 2015. The red triangles in the lower right panel indicate actual cycle slips. The phase ionospheric-free, i.e., $L_{\mathrm{IF}}$, residuals are obtained by introducing only one ambiguity parameter for satellite $\mathrm{C} 04$ when performing the PPP processing
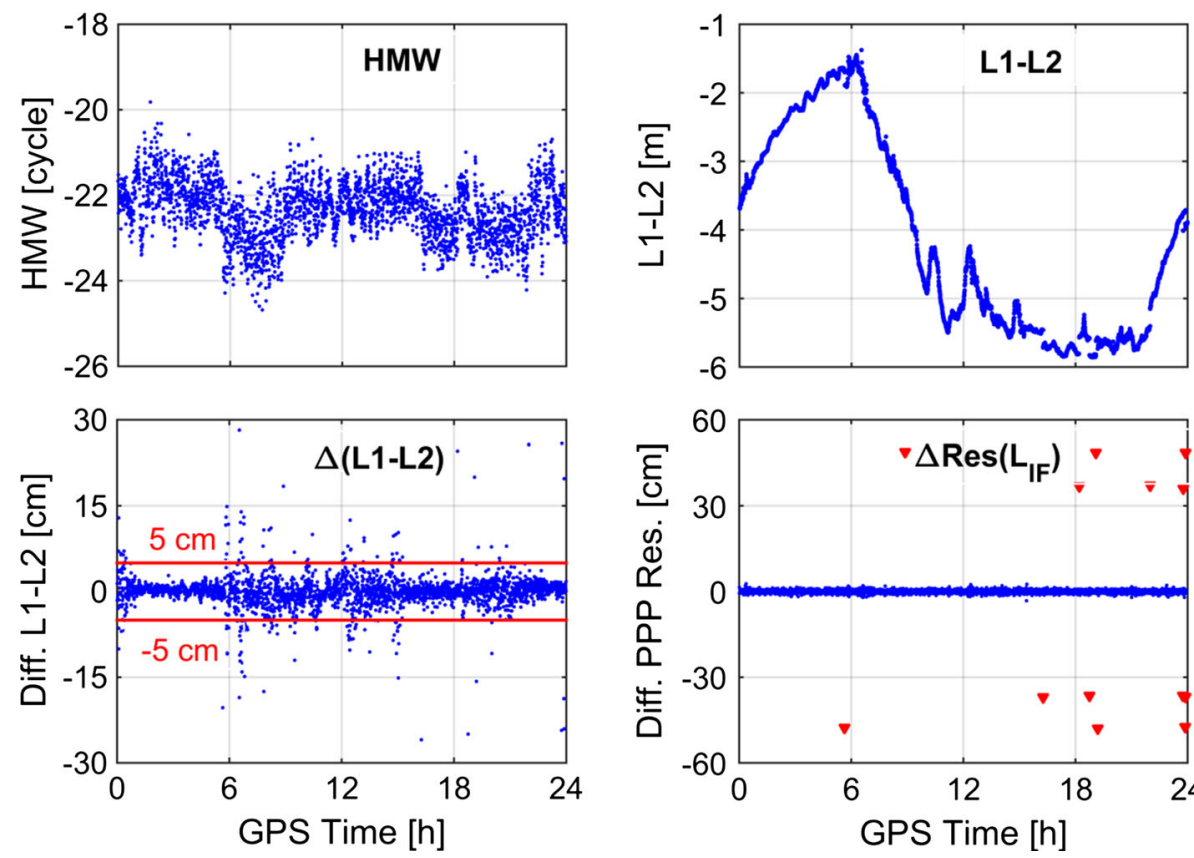

found by $\Delta(\mathrm{L} 1-\mathrm{L} 2)$ can be reflected in the series of $\Delta L_{\mathrm{GIF}}$. For example, some cycle slips with the value of $(1,1)$ and $(-1,-1)$ between 14 and $21 \mathrm{~h}$ cannot be reflected by $\Delta L_{\mathrm{GIF}}$ due to the cycle slip insensitive combinations $(1,1$, $1)$ and $(-1,-1,-1)$ of the triple-frequency $L_{\mathrm{GIF}}$ combination.

The L1-L2 combination is sensitive to small cycle slips and has the potential to detect those 1-cycle slips occurring on both frequencies simultaneously. However, such phaseonly combination would probably be affected by the ionospheric variations. When the changes of ionospheric delay are slow and smooth, such 1-cycle slips can be easily detected by a first-order difference method. While ionospheric scintillation happens, the intensity fluctuation of the ionospheric delay in L1-L2 cannot be removed completely by a first-order or even high-order difference method.

According to Ji et al. (2013), the ionospheric condition around the polar area is often active. Figure 2 illustrates the cycle slips in BDS C04 carrier phase observations, which are collected at Antarctic station CAS1 with 30-s sampling rate over a period of $24 \mathrm{~h}$. On the one hand, the HMW combination series reveal these cycle slips are small and hard to be detected by the traditional TurboEdit algorithm. On the other hand, the intensive fluctuations in L1-L2 series cannot be removed by a simple difference method. Given a tight detection threshold, such as $5 \mathrm{~cm}$, cycle slips and residual ionospheric variations are hard to be distinguished from the series of $\Delta(\mathrm{L} 1-\mathrm{L} 2)$. In that case, a high false-alarm rate will be observed, which can be further confirmed by the differenced phase ionospheric-free residuals of PPP. In addition, it is worth to mention that a triple-frequency-based method is not applicable in this example because the station
CAS1 does not support B3 frequency tracking for BDS. So, how to model the effect of ionospheric delay in the L1-L2 combination is crucial for cycle slip detection under such active ionospheric conditions.

\section{Enhanced cycle slip detection using dual-frequency phase geometry-free combination}

Without loss of generality, we assume in the following analysis that receivers can track B1 $(1561.098 \mathrm{MHz})$ and B2 (1207.14 MHz) frequencies. Ignoring the high-order contributions of ionospheric path delays, the dual-frequency geometry-free phase combination at epoch $t_{i}$ can be written as

$L_{\mathrm{GF}}\left(t_{\mathrm{i}}\right)=(\alpha-1) I\left(t_{\mathrm{i}}\right)+\lambda_{1} A_{1}\left(t_{\mathrm{i}}\right)-\lambda_{2} A_{2}\left(t_{\mathrm{i}}\right)+e_{12}\left(t_{\mathrm{i}}\right)$

where $\alpha=f_{1}^{2} / f_{2}^{2}$ is the squared ratio of B1 to B2 frequencies, $I\left(t_{i}\right)$ is the ionospheric range delay on B1 signal, $\lambda_{1} \approx 19.2 \mathrm{~cm}$ and $\lambda_{2} \approx 24.8 \mathrm{~cm}$ are the wavelengths of B1 and B2 signals, $A_{1}\left(t_{i}\right)$ and $A_{2}\left(t_{i}\right)$ are carrier phase ambiguities of $\mathrm{B} 1$ and $\mathrm{B} 2$ in units of cycles, and $e_{12}\left(t_{i}\right)$ is the difference of phase noise between B1 and B2 which is supposed to be independent and identically distributed by $N\left(0, \sigma_{0}^{2}\right)$.

The term $\lambda_{1} A_{1}(t)-\lambda_{2} A_{2}(t)$ will be maintained as a constant over time, unless a cycle slip occurs. The ionospheric delay $I(t)$ mainly depends on the total electron content (TEC) along the path of signal propagation. When the ionosphere is quiet, $I(t)$ can be treated as a low-frequency signal and varies smoothly over time (Banville and Langley 2013). However, this feature will no longer be 
kept as soon as ionospheric scintillation happens. The ionospheric disturbances scatter the signal in random directions and generate a rapid fluctuation superposed on the low-frequency signal caused by the TEC variations. In order to accommodate the case of ionospheric scintillation, we treat $\left\{L_{\mathrm{GF}}(t)\right\}$ as a nonstationary time series. A polynomial fit $Q$ to $(\alpha-1) I$ is first subtracted from $\left\{L_{\mathrm{GF}}(t)\right\}$, and then discontinuities are searched in the residuals $R \triangleq L_{G F}-Q$. Particularly, the heteroscedasticity of the residual series caused by ionospheric variations is modeled to determine the threshold for cycle slip detection adaptively.

\section{Detrending of the time series of $\left\{L_{\mathrm{GF}}(t)\right\}$}

Unlike the TurboEdit algorithm (Blewitt 1990), we extract the trend $Q$ of $(\alpha-1) I$ from the geometry-free phase combinations directly rather than the pseudorange combinations $P_{2}-P_{1}$. A good detrending method is necessary for detecting small cycle slips, especially for the case of 1-cycle slip occurring on both B1 and B2 frequencies simultaneously. On the other hand, the fit residuals $R$ can be used to infer the size of individual cycle slips subsequently.

The difficulty of using phase data to carry out the polynomial fit of $(\alpha-1) I$ lies on how to avoid the effect of cycle slips. A natural idea is to use the differenced series $\left\{\Delta L_{\mathrm{GF}}(t)\right\}$ between two consecutive epochs instead of $\left\{L_{\mathrm{GF}}(t)\right\}$ to control the influence of cycle slips on limited data points, and then design a robust fit algorithm to estimate the unknown polynomial coefficients. Given a short interval, which is typical $10-15 \mathrm{~min}$, a quadratic or cubic polynomial will satisfy the requirement of detrending. Thus, a low-order piecewise polynomial fit strategy will be applied in this study.

Considering the robust polynomial fit on subinterval $\left[t_{s}\right.$, $\left.t_{e}\right]$, we denote $Q_{i}(\boldsymbol{\beta}) \triangleq \beta_{0}+\sum_{j=1}^{m} \beta_{j}\left(t_{i}-t_{\mathrm{s}}\right)^{j}, \Delta Q_{i}(\boldsymbol{\beta}) \triangleq Q_{i}$ $(\boldsymbol{\beta})-Q_{i-1}(\boldsymbol{\beta})$ and $y_{i} \triangleq L_{\mathrm{GF}}\left(t_{i}\right)-L_{\mathrm{GF}}\left(t_{i-1}\right)$, where $t_{i} \in$ $\left[t_{s}, t_{e}\right], i=1,2, \ldots, N$. The robust estimator of $\boldsymbol{\beta}$ is defined as follows

$\min \left\{(\boldsymbol{y}-\Delta Q(\boldsymbol{\beta}))^{\mathrm{T}} \boldsymbol{P}_{R}(\boldsymbol{y}-\Delta Q(\boldsymbol{\beta}))\right\}$

where $\boldsymbol{P}_{R}=\mathbf{W} \Sigma^{-1} \mathbf{W}$ is formed by the inverse of the covariance matrix $\Sigma=\operatorname{Var}\left(\Delta \boldsymbol{e}_{12}\right)$ and the robust weight matrix $\mathbf{W}=\operatorname{diag}\left(w_{1}, w_{2}, \ldots, w_{N}\right)$. It should be mentioned that the value of $\beta_{0}$ does not affect the minimization of (2) since it has been canceled by $\Delta Q(\boldsymbol{\beta})$. However, to ensure the adjacent polynomials are connected, the value of $\beta_{0}$ should be predetermined by the last fit value in previous subinterval.

According to the former assumption that $\boldsymbol{e}_{12} \sim N\left(0, \sigma_{0}^{2} \mathbf{I}\right), \Sigma$ can be easily computed by
$\Sigma_{i j}= \begin{cases}2 \sigma_{0}^{2}, & i=j \\ -\sigma_{0}^{2}, & |i-j|=1 \\ 0, & |i-j|>1\end{cases}$

The $i$ th diagonal element of the robust weight matrix $\mathbf{W}$ is determined by

$w_{i}= \begin{cases}1, & \left|y_{i}-\Delta Q_{i}(\boldsymbol{\beta})\right| \leq 4 \hat{\sigma} \\ 0, & \left|y_{i}-\Delta Q_{i}(\boldsymbol{\beta})\right|>4 \hat{\sigma}\end{cases}$

where $\hat{\sigma}$ is an estimate of standard deviation of the fit residuals.

For normally distributed data,

$\hat{\sigma}=1.483 \operatorname{med}\left(\left|y_{i}-\Delta Q_{i}(\boldsymbol{\beta})\right|\right)$

is often employed as a consistent estimator of the standard deviation $\sigma$ (Rousseeuw and Croux, 1993; Xu, 2005), where $\operatorname{med}\left(\left|y_{i}-\Delta Q_{i}(\boldsymbol{\beta})\right|\right)$ is the median of the absolute values of residual series $\left\{\left|y_{i}-\Delta Q_{i}(\boldsymbol{\beta})\right|\right\}$.

To solve the minimization problem of (2), the weighted least squares estimator needs to be applied iteratively. Knowing that the value of $\left|y_{i}\right|$ is often extremely large or small compared to the normal points if a cycle slip occurs at epoch $t_{i}$, the largest $40 \%$ and smallest $10 \%$ data of $\left\{\left|y_{i}\right|\right\}$ are empirically excluded to start the robust fit procedure. The iterative algorithm is described as follows:

Step 1 Initialization: $k=0,50 \%$ data are used to initialize $\mathbf{W}^{(0)}$ and $\boldsymbol{\beta}^{(0)}$

Step 2 Compute residual series $\left\{y_{i}-\Delta Q_{i}\left(\boldsymbol{\beta}^{(k)}\right)\right\}$ as well as the standard deviation $\hat{\sigma}^{(k)}$ according to (5)

Step 3 Update the weight matrix $\mathbf{W}^{(k+1)}$ by (4) and solve $\boldsymbol{\beta}^{(k+1)}$ from (2) by the weighted least squares

Step 4 Justify whether $\mathbf{W}^{(k+1)}=\mathbf{W}^{(k)}$, if so, stop the iteration; otherwise, let $k=k+1$ and go to step 2.

Figure 3 demonstrates the performance of the robust polynomial fit algorithm using the phase geometry-free combinations. A quadratic polynomial fit is applied on each 10-min subinterval. As can be seen from the figure, the long-term trend (the red curve) of the ionospheric variations can be effectively extracted, though some discontinuities appeared in the original series of $\left\{L_{\mathrm{GF}}(t)\right\}$ (the blue curve). Then, cycle slips can be easily recognized by the polynomial fit residual series (the black curve).

\section{Statistical analysis of the robust fit residuals}

The differenced fit residuals $\Delta R_{i}$ between two consecutive epochs will be used as a basic test quantity in our enhanced cycle slip detection method. In particular, each $\Delta R_{i}$ is considered as a random variable with non-constant mean 

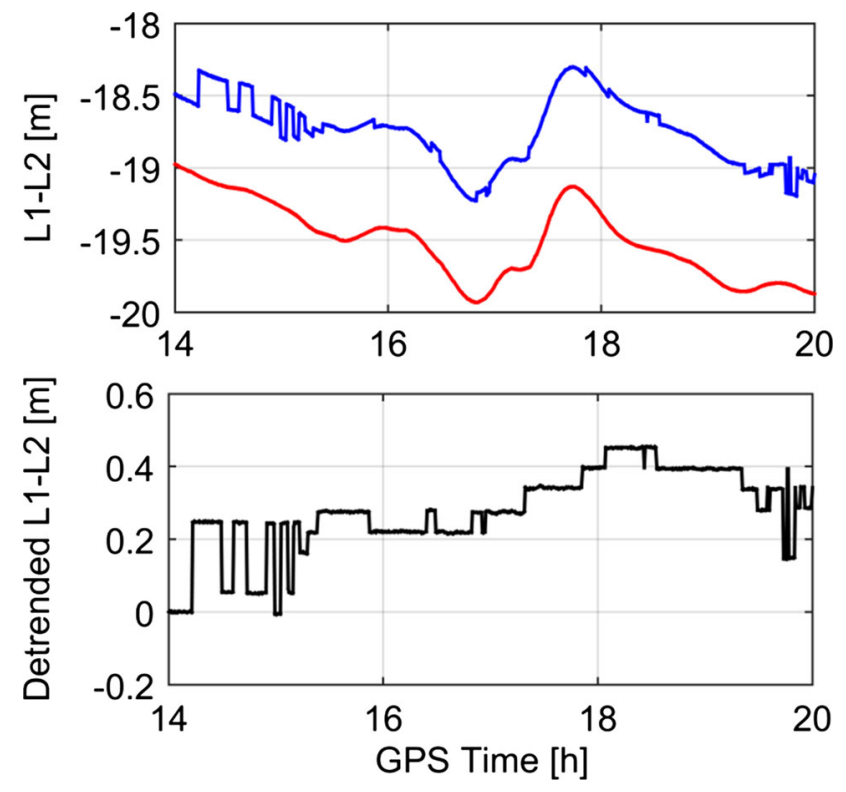

Fig. 3 Performance of the robust polynomial fit algorithm using the phase geometry-free combinations of satellite $\mathrm{C} 05$ tracked by station JFNG on September 1, 2015. The original L1-L2 series (blue) and the shifted robust polynomial fit curve (red) are given in the top panel. The detrended L1-L2 series, i.e., the polynomial fit residuals (black), are given in the bottom panel

$\left(\lambda_{1} \delta N_{1}-\lambda_{2} \delta N_{2}\right)$, which is different from zero when cycle slip occurs, and with variance depending on both phase noises and residual ionospheric variations between two consecutive epochs. The variance of $\Delta R_{i}$ is essential for determining a reliable threshold for cycle slip detection. In this section, we present the statistical characteristics of $\Delta R_{i}$ under both quiet and active ionospheric conditions. The outlier points whose corresponding robust weights are equal to zero have been removed temporarily before statistical analysis.

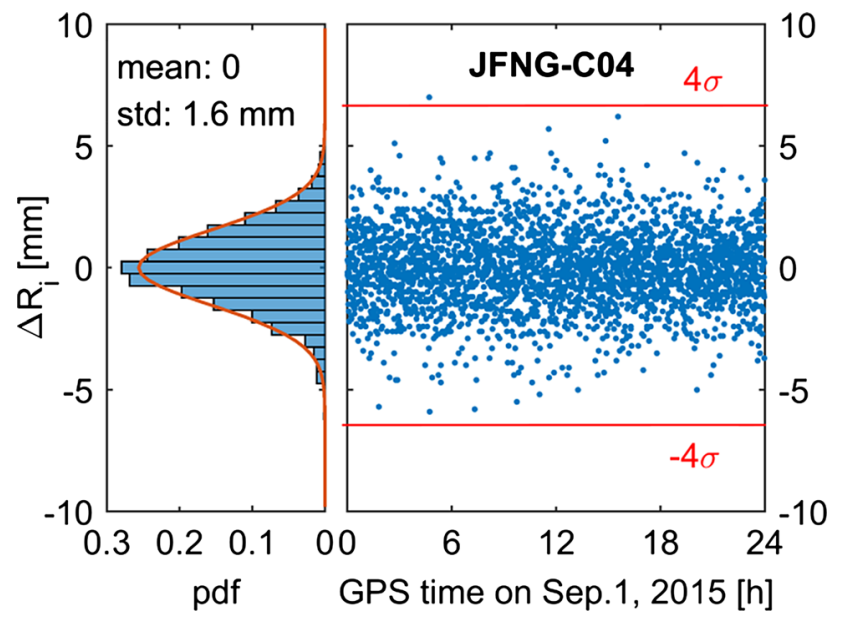

Fig. 4 Statistical characteristics of $\left\{\Delta R_{i}\right\}$ under quiet ionospheric condition
Figure 4 shows a typical example of the differenced fit residuals of $\left\{L_{\mathrm{GF}}(t)\right\}$ under quiet ionospheric condition. The histogram shown in the left panel indicates that $\Delta R_{i}$ is well normally distributed with mean zero and $\sigma$ of about $1.6 \mathrm{~mm}$. In other words, the distribution of each random variable $\Delta R_{i}$ is homoscedastic. Thus, a uniform threshold of $4 \sigma$ can be used to justify whether a cycle slip occurs.

Figure 5 shows an example of the differenced fit residuals of $\left\{L_{\mathrm{GF}}(t)\right\}$ under active ionospheric condition. As shown in the right panel, the variance of $\left\{\Delta R_{i}\right\}$ is much larger than that under quiet ionospheric condition. The zero-mean time series $\left\{\Delta R_{i}\right\}$ exhibits significant volatility clustering. On the other hand, the histogram shows a character of leptokurtosis and fat-tail compared with the estimated normal density function. In this case, using a uniform threshold of $4 \sigma$ will induce a high falsealarm rate.

The fluctuation of residual ionospheric variations leads to the volatility clustering in the differenced fit residual series $\left\{\Delta R_{i}\right\}$. In fact, $\left\{\Delta R_{i}\right\}$ is a typical conditional heteroskedastic time series, which allows the conditional variance to change over time and leave the unconditional variance constant. Apparently, the conditional variance is more appropriate to describe actual uncertainty of each random variable $\Delta R_{i}$. In order to determine a more reliable threshold for cycle slip detection, we should take into account such heteroscedasticity rather than use a uniform threshold over the whole arc.

\section{GARCH modeling for the time series of $\left\{\Delta R_{i}\right\}$}

The generalized autoregressive conditional heteroscedastic (GARCH) model (Bollerslev 1986) is appropriate for time series that exhibits significant volatility clustering.

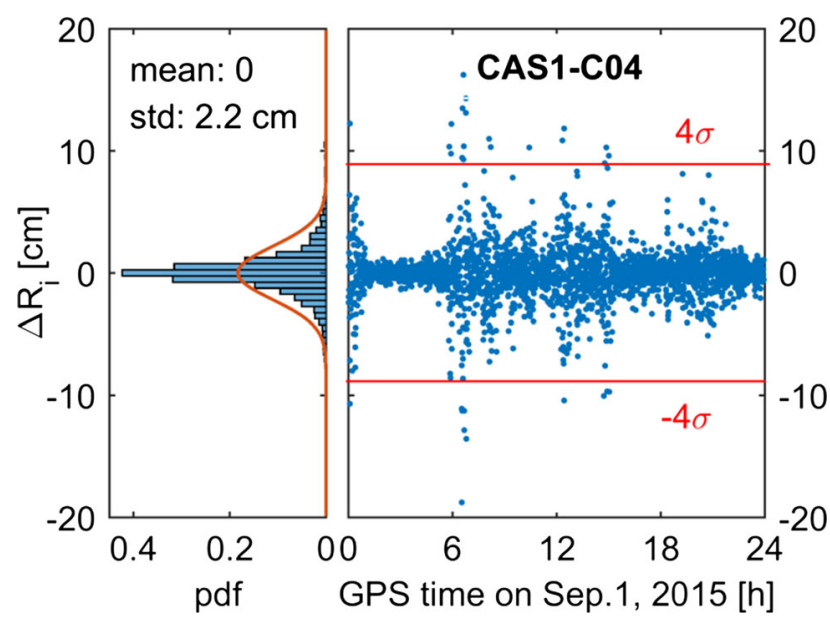

Fig. 5 Statistical characteristics of $\left\{\Delta R_{i}\right\}$ under active ionospheric condition 
Particularly, the simplest case of GARCH $(1,1)$ can satisfy the requirement of conditional variance modeling for the series of $\left\{\Delta R_{i}\right\}$, according to our extensive numerical analysis.

Assume that the conditional distribution of $\Delta R_{i}$ satisfies $\Delta R_{i} \mid H_{i-1} \sim N\left(0, \sigma_{i}^{2}\right)$, and then the conditional variance $\sigma_{i}^{2}$ is defined as

$\sigma_{i}^{2}=\operatorname{Var}\left(\Delta R_{i} \mid H_{i-1}\right)$

where $H_{i-1}$ is the history of the process, including both the past conditional variances $\sigma_{1}^{2}, \sigma_{2}^{2}, \ldots, \sigma_{i-1}^{2}$ and the past innovations $\Delta R_{1}, \Delta R_{2}, \ldots, \Delta R_{i-1}$.

The $\operatorname{GARCH}(1,1)$ model formulates $\sigma_{i}^{2}$ as

$\sigma_{i}^{2}=\omega+\alpha_{1} \Delta R_{i-1}^{2}+\beta_{1} \sigma_{i-1}^{2}$

where $\omega, \alpha_{1}$ and $\beta_{1}$ are unknown parameters that can be estimated by the iterative maximum likelihood method (Bollerslev 1986). Keep in mind that the gross outlier points, whose corresponding weights are equal to zero in the robust polynomial fit processing, should not be used to estimate the parameters in the GRACH $(1,1)$ model. The structure of (7) allows the conditional heteroscedastic model to possess adaptive learning mechanism from the histories. Particularly, the conditional variance $\sigma_{i}^{2}$ tends to be homoscedastic when $\alpha_{1} \rightarrow 0$ and $\beta_{1} \rightarrow 0$. So such model can be applied to any cases no matter whether the ionospheric conditions are active or not.

Using conditional variance instead of sample variance of the series $\left\{\Delta R_{i}\right\}$ allows us to change the threshold for cycle slip detection adaptively. Figure 6 demonstrates the comparison of conditional variance and sample variance of $\left\{\Delta R_{i}\right\}$ under different ionospheric conditions. The top panel shows the case of quiet ionospheric condition under which the estimated conditional variance and sample variance are approaching. Both conditional variance and sample variance can be used to determine the threshold for cycle slip detection with high sensitivity. The middle panel shows the case of partial ionospheric scintillation over a specified period. The ionosphere is quiet over the entire day expect the duration between 12:00 and 18:00 (GPST). In this case, using the sample variance to determine the threshold for cycle slip detection will lead to a high falsealarm rate during the period of ionospheric scintillation. The bottom panel shows the case of ionospheric scintillation over the whole day. The volatility clustering of the series $\left\{\Delta R_{i}\right\}$ can be well captured by the conditional variance. Using the conditional variance in cycle slip detection can largely decrease the false-alarm rate when ionospheric fluctuation is intensive. On the other hand, the sensitivity to small cycle slips can still be retained when the ionosphere is quiet.
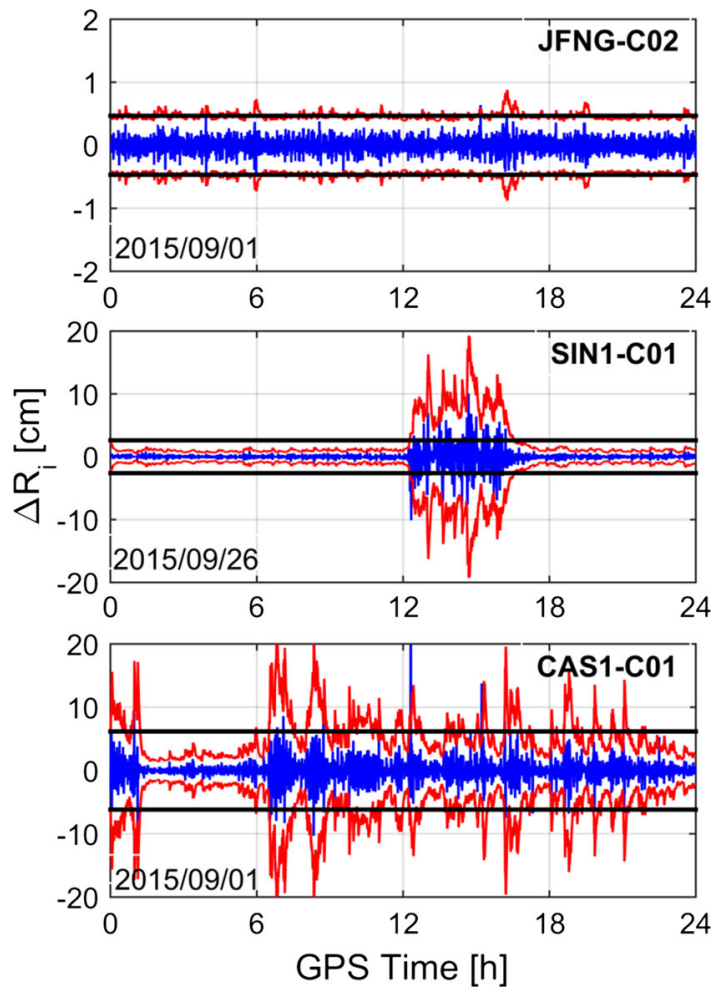

Fig. 6 Comparison of detection threshold between conditional variance (red) and sample variance (black) of $\left\{\Delta R_{i}\right\}$ (blue) under different ionospheric conditions

\section{Numerical results and analysis}

Our complete cycle slip detection and repair strategy comprise 3 steps. (1) Conduct the enhanced cycle slip detection and divide the data into sub-arcs. (2) Conduct cycle slip detection using the HMW combination on an individual sub-arc to further recognize those insensitive cycle slip pairs to the phase geometry-free combination. (3) Perform cycle slip repair by solving the equations

$\left\{\begin{array}{c}\delta N_{1}-\delta N_{2}=\Delta A_{i} \\ \lambda_{1} \delta N_{1}-\lambda_{2} \delta N_{2}=\Delta R_{i}\end{array}\right.$

where $\Delta A_{i}$ is the difference of mean value of the HMW combinations between two consecutive arcs, which has been rounded to an integer value. The integer values of $\left(\delta N_{1}, \delta N_{2}\right)$ are determined by rounding the float solutions $\left(\delta \hat{N}_{1}, \delta \hat{N}_{2}\right)$ to the nearest integers. The recognized cycle slip can be repaired successfully only if the fractional parts of $\left(\delta \hat{N}_{1}, \delta \hat{N}_{2}\right)$ are within a predefined threshold.

The capability of the enhanced cycle slip detection method has been tested by both simulated and real cycle slips for BeiDou GEO carrier phase observations. All of the datasets were collected by MGEX stations at an interval of $30 \mathrm{~s}$. The performance of real cycle slip detection and 
Table 2 Effect of simulated cycle slips on the HMW combination, the L1-L2 combination and the TECR computed under different sampling rate

\begin{tabular}{|c|c|c|c|c|c|}
\hline \multirow{2}{*}{$\begin{array}{l}\text { Cycle slip on B1 } \\
\text { (cycle) }\end{array}$} & \multirow{2}{*}{$\begin{array}{l}\text { Cycle slip on B2 } \\
\text { (cycle) }\end{array}$} & \multirow{2}{*}{$\begin{array}{l}\text { Effect on HMW } \\
\text { (cycle) }\end{array}$} & \multirow{2}{*}{$\begin{array}{l}\text { Effect on } \\
\text { L1-L2 (cm) }\end{array}$} & \multicolumn{2}{|c|}{ Effect on TECR (TECU/s) } \\
\hline & & & & $\Delta t=1 \mathrm{~s}$ & $\Delta t=30 \mathrm{~s}$ \\
\hline 1 & 0 & 1 & 19.20 & 1.727 & 0.058 \\
\hline 0 & 1 & -1 & -24.83 & -2.234 & -0.074 \\
\hline 1 & 1 & 0 & -5.63 & -0.506 & -0.017 \\
\hline 4 & 3 & 1 & 2.31 & 0.208 & 0.007 \\
\hline 5 & 4 & 1 & -3.32 & -0.299 & -0.010 \\
\hline-9 & -7 & -2 & 1.01 & 0.091 & 0.003 \\
\hline
\end{tabular}

repair will be demonstrated under both quiet and active ionospheric conditions.

\section{Simulated cycle slip detection}

Since cycle slips that satisfy $\left|\delta N_{1}-\delta N_{2}\right| \geq 3$ cycles can be generally detected by the HMW combinations, we only focus on the detectability of those small cycle slips subject to $\left|\delta N_{1}-\delta N_{2}\right| \leq 2$ cycles in simulation analysis. According to the description above, frequent 1-cycle slips often occur in low-elevation BeiDou GEO carrier phase observations. Thus, a sequence of 1-cycle slips on B1 or/and B2 frequencies are simulated to evaluate our proposed method. In addition, three other cycle slip pairs $(4,3),(5,4)$ and $(-9$, -7) are also tested due to their insensitivities to the phase geometry-free combination (Cai et al. 2013).

Table 2 shows the effect of simulated cycle slip pairs on the HMW combination, the L1-L2 combination and the TECR under different sampling rate, respectively. As can be seen from the rightmost column of the table, the TECR method will be invalid to all simulated cycle slips under a sampling rate of $30 \mathrm{~s}$ if one employs a fixed threshold of $0.15 \mathrm{TECU} / \mathrm{s}$ (Liu 2011).

To demonstrate the sensitivity of the proposed method to small cycle slips, a total of 18 artificial cycle slips have been introduced to a piece of "clean" carrier phase observations of satellite $\mathrm{C} 02$, which is tracked by station JFNG on September 1, 2015, between 10:00 and 14:00 (GPST).

First, the deficiencies of the HMW combination to detect these simulated slips are shown in Fig. 7. As noted above, the HMW combination is insensitive to cycle slips that are identical on both frequencies, such as the one occurred at 11:00 (GPST). On the other hand, missing detection for the HMW combination is probably encountered when small cycle slips frequently occur in noisy situations. For example, failure of detection for the cycle slip $(-1,0)$ at 11:35 (GPST) leads to the missing detection for all subsequent cycle slips. As a result, only 3 of the 18 simulated cycle slips can be recognized using the HMW

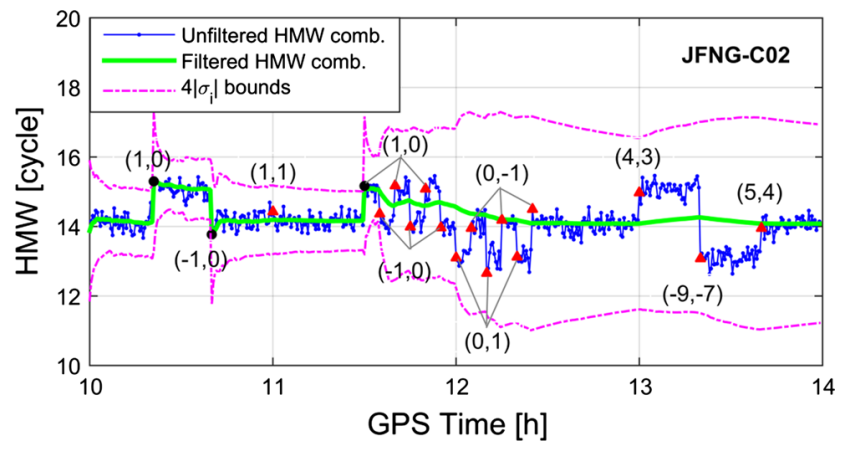

Fig. 7 Performance of the HMW combination for simulated cycle slips. The black dots indicate cycle slips that can be detected and red triangles indicate cycle slips that cannot be detected

combination. Since the effect of all simulated cycle slips on L1-L2 combination as shown in Table 2 is within the threshold $(6 \times 5.63 \mathrm{~cm})$ suggested by Blewitt $(1990)$, the ionospheric combinations cannot further recognize those undetected cycle slips. Therefore, the TurboEdit algorithm experiences a high missing alarm rate in this simulation.

Figure 8 shows the performance of our proposed detection method for simulated cycle slips. As shown in the top panel, all such small cycle slips can be recognized by the enhanced detection method, even for those insensitive cycle slip pairs, such as $(4,3),(5,4)$ and $(-9,-7)$. It should be emphasized that the most challenging cycle slip combination $(-9,-7)$ cannot be recognized by any traditional methods based on time-differenced geometry-free phase combinations due to the effect on L1-L2 approaching the level of normal ionospheric variations. Since we employ the series of detrended phase geometry-free combinations, the variation caused by the ionosphere can be completely removed from the L1-L2 combination when the ionospheric condition is quiet enough. Thus, this challenging cycle slip pair can be recognized successfully by the proposed method in this simulation. The bottom panel shows the performance of cycle slip detection on individual sub-arcs using the HMW combinations. Since all simulated cycle slips have been recognized by the 

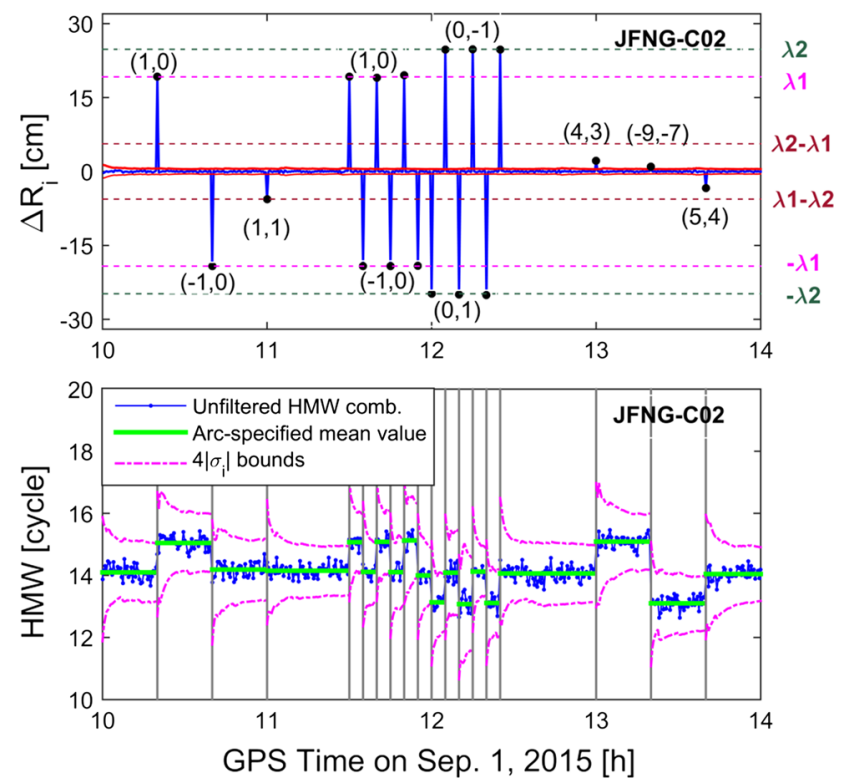

Fig. 8 Performance of the proposed cycle slip detection method in simulation. In the top panel, the black dots indicate cycle slips that have been recognized. In the bottom panel, the vertical lines indicate the beginning of a new sub-arc determined by the previous enhanced cycle slip detection method

previous enhanced detection method, the further detection in this simulation is mainly used as a complement to provide information for cycle slip repair subsequently. However, it should be mentioned that the detectability of the enhanced method depends on the level of the robust polynomial fit residuals. Missing detection may be encountered while intense ionospheric disturbances happened, especially for the cycle slip pairs $(-9,-7)$ and $(5$, 4). Thus, a further check on individual sub-arcs using the HMW combinations is necessary to ensure the reliability for cycle slip detection.

\section{Real cycle slip detection under quiet ionospheric condition}

The performance of the proposed method under quiet ionospheric condition is demonstrated by satellite $\mathrm{C} 05$, which is tracked at mid-latitude MGEX station JFNG. Figure 9 illustrates the detectability of the enhanced method for small cycle slips. Isolated outliers have been removed before cycle slip detection. As shown in the figure, all small cycle slips can be detected, even for the 1cycle slip occurring on both B1 and B2 frequencies. In addition, most of the values of $\Delta R_{i}$ at cycle slip epochs approximate the theoretical values $\left(\lambda_{1} \delta N_{1}-\lambda_{2} \delta N_{2}\right)$, which indicate the effectiveness of our robust polynomial fit algorithm.

On September 1, 2015, a total of 108 cycle slips have been detected for satellite C05 at station JFNG. Figure 10

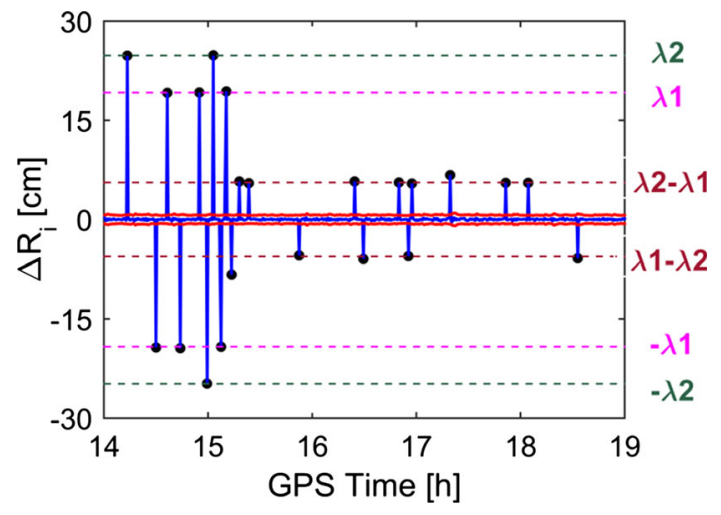

Fig. 9 Performance of the enhanced cycle slip detection method for satellite C05 tracked by station JFNG on September 1, 2015. The two red curves depict the adaptive detection threshold determined by the GRACH $(1,1)$ model. The black dots indicate cycle slips detected by the proposed method

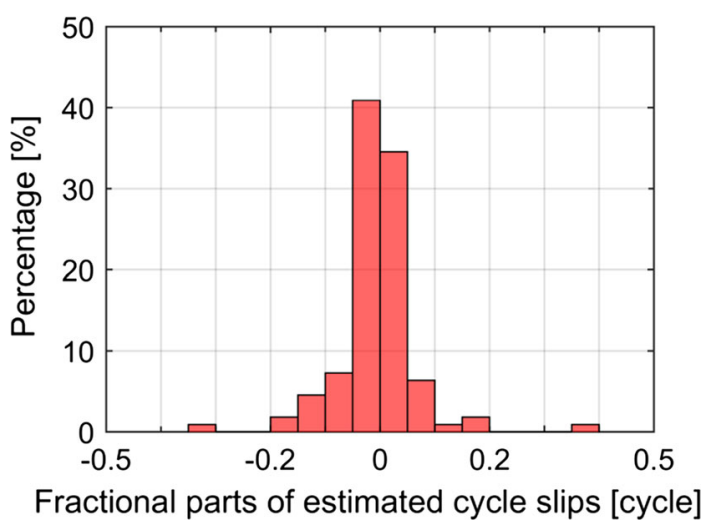

Fig. 10 Distribution of the fractional parts after rounding the estimated float cycle slips of satellite C05 tracked by station JFNG on September 1, 2015

shows the distribution of the fractional parts after rounding the estimated float cycle slips to the nearest integers. About $78 \%$ of the fractional parts are within \pm 0.1 cycles. More than $98 \%$ of the fractional parts of the float cycle slips are within \pm 0.2 cycles. Therefore, most of the detected cycle slips can be repaired successfully given a threshold of 0.2 cycles. Among the 106 repaired cycle slips, 35 1-cycle slips are occurring on B1 frequency, 36 1-cycle slips on B2 frequency, and 35 1-cycle slips on both B1 and B2 frequencies simultaneously. Such extensive series of 1-cycle slips presents great challenges to existing dual-frequency cycle slip detection methods but can be recognized using our proposed method.

\section{Real cycle slip detection under active ionospheric condition}

Cycle slip detection under active ionospheric condition using the dual-frequency phase geometry-free combination 


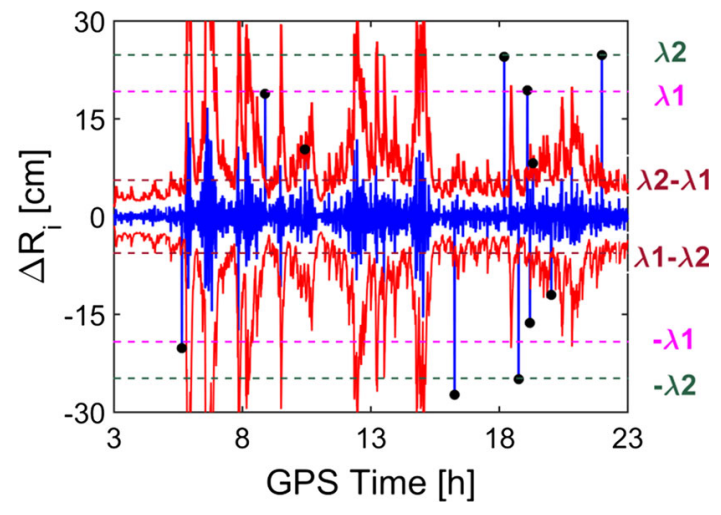

Fig. 11 Performance of the enhanced cycle slip detection method for satellite C04 tracked by station CAS1 on September 1, 2015. The two red curves depict the adaptive detection threshold determined by the GRACH $(1,1)$ model. The black dots indicate cycle slips that have been detected including false alarms

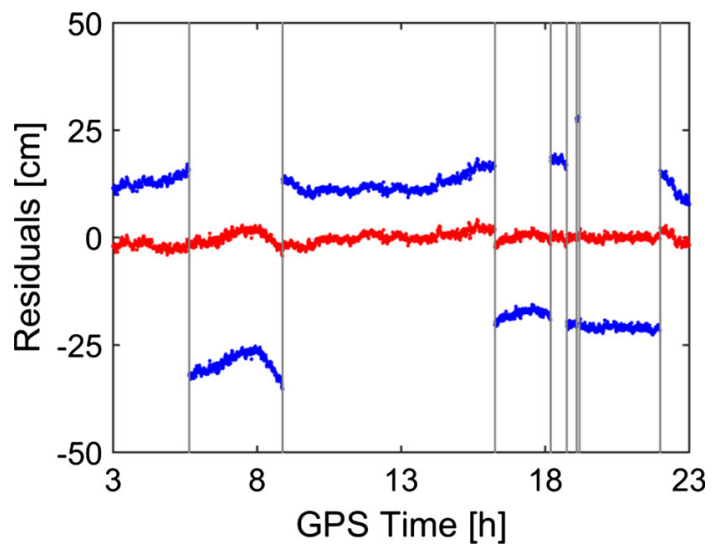

Fig. 12 Phase ionospheric-free residuals of PPP before (blue) versus after ( $r e d$ ) recognizing cycle slips for satellite $\mathrm{C} 04$ tracked by station CAS1 on September 1, 2015. The vertical lines indicate cycle slip epochs has been a challenge for many years, especially for the case with a low data sampling rate. The difficulty lies on how to determine a reliable detection threshold, which allows recognizing as many cycle slips as possible and yet not introduce too many false alarms. Unlike other L1-L2 based methods, an adaptive detection threshold can be obtained by a conditional heteroscedastic model for the proposed method. In that case, the detectability for a specific epoch can be automatically adjusted according to the local intensity of ionospheric variations.

Figure 11 demonstrates the performance of our proposed method for satellite $\mathrm{C} 04$ tracked at Antarctic station CAS1, where the ionospheric condition is active all day long. Actual cycle slips can be identified by the phase ionospheric-free residuals of PPP processing, if only one ambiguity parameter was introduced and solved for satellite C04. As can be seen from Fig. 12, a total of eight cycle slips occurred between 3:00 and 23:00 (GPST) on September 1, 2015. As shown in Fig. 11, all of these cycle slips can be successfully detected and only 3 false alarms appeared using the proposed method. In Fig. 12, those unexpected jumps in phase ionospheric-free residuals almost vanished after the cycle slips have been recognized.

It should be mentioned that missing detection is always more serious than a few false alarms in cycle slip detection. We also evaluated the performance of the TurboEdit algorithm used to the same data set. Results indicate that the TurboEdit algorithm cannot detect most of these small cycle slips due to the effect of large code multipath errors on the HMW combination.

Table 3 shows the repair information for the detected cycle slips in Fig. 11. Since the precision of $\Delta R_{i}$ depends on the performance of polynomial fit to the local ionospheric variations, the temporal resolution of $30 \mathrm{~s}$ is

Table 3 Cycle slip repair information for satellite C04 tracked by station CAS1 on September 1, 2015

\begin{tabular}{|c|c|c|c|c|c|c|}
\hline \multirow[t]{2}{*}{ Epoch (hh:mm:ss) } & \multirow[t]{2}{*}{$\Delta R_{i}(\mathrm{~cm})$} & \multirow[t]{2}{*}{$\Delta \mathrm{HMW}_{i}($ cycle $)$} & \multirow[t]{2}{*}{$\Delta A_{i}($ cycle $)$} & \multicolumn{3}{|l|}{$\left(\delta N_{1}, \delta N_{2}\right)$} \\
\hline & & & & Float solution & Integer solution & Fractional part \\
\hline 05:38:30 & -20.2 & -1.05 & -1 & $(-0.82,0.18)$ & $(-1,0)$ & 0.18 \\
\hline 08:53:00 & 18.9 & 0.89 & 1 & $(1.06,0.06)$ & $(1,0)$ & 0.06 \\
\hline 10:26:00 & 10.3 & 0.03 & $\mathbf{0}$ & $(-1.83,-1.83)$ & $(-2,-2)$ & 0.17 \\
\hline $16: 15: 30$ & -27.3 & -0.71 & -1 & $(0.44,1.44)$ & $(0,1)$ & 0.44 \\
\hline 18:11:30 & 24.5 & 0.83 & 1 & $(0.06,-0.94)$ & $(0,-1)$ & 0.06 \\
\hline 18:45:00 & -25.0 & -0.99 & -1 & $(0.02,1.02)$ & $(0,1)$ & 0.02 \\
\hline 19:05:30 & 19.4 & 1.25 & 1 & $(0.97,-0.03)$ & $(1,0)$ & -0.03 \\
\hline 19:11:30 & -16.3 & -0.97 & -1 & $(-1.52,-0.52)$ & $(-2,-1)$ & 0.48 \\
\hline 19:18:30 & 8.2 & -0.07 & $\mathbf{0}$ & $(-1.46,-1.46)$ & $(-1,-1)$ & -0.46 \\
\hline 20:01:30 & -12.0 & -0.13 & $\mathbf{0}$ & $(2.13,2.13)$ & $(2,2)$ & 0.13 \\
\hline 21:59:30 & 24.8 & 0.77 & 1 & $(0.01,-0.99)$ & $(0,-1)$ & 0.01 \\
\hline
\end{tabular}

False-alarm epochs have been marked in bold 
probably insufficient under active ionospheric condition. Thus, a wrong estimation of $\left(\delta N_{1}, \delta N_{2}\right)$ may be encountered, such as the cycle slip at 19:11:30 (GPST). Due to the large fractional part $(0.48$ cycles $)$ of the float solution, this cycle slip can only be detected but not be repaired. On the other hand, a more rigorous threshold, such as 0.1 cycles, for cycle slip repair under active ionospheric condition is strongly recommended to avoid incorrect repair events. In summary, the objective of reliable cycle slip detection under active ionospheric condition can be achieved by the proposed method. As to cycle slip repair, a high data sampling rate may be helpful to improve the success rate. This is, however, beyond the scope of this research.

\section{Conclusions}

Extensive 1-cycle slips have been found in low-elevation BeiDou GEO carrier phase observations, which are collected by the MGEX tracking stations. One should pay more attention to such contaminated data when using them in PPP and POD processing. We proposed an enhanced cycle slip detection method based on the series of dualfrequency phase geometry-free combinations. To deal with the effect of ionospheric variation, the long-term trend is first removed from the L1-L2 series by a robust polynomial fit algorithm. Then, the fit residuals are treated as a nonstationary and heteroskedastic time series. The GARCH $(1,1)$ model is employed to estimate the timevariant conditional variance, which will be used as an adaptive threshold in cycle slip detection subsequently. Simulated and real data tests reveal that most of the 1-cycle slips can be detected and repaired successfully by the proposed method. The proper modeling for ionospheric variation even allows us to detect small cycle slips in the case of ionospheric scintillation.

Acknowledgements This research was supported by the National Natural Science Foundation of China (Nos. 61573367, 61370013, and 91438202) and the international GNSS Monitoring \& Assessment System (iGMAS) data center. The authors would like to thank the IGS Multi-GNSS Experiment (MGEX) project for providing the GNSS data used in this research.

\section{References}

Banville S, Langley RB (2013) Mitigating the impact of ionospheric cycle slips in GNSS. J Geod 87(2):179-193. doi:10.1007/ s00190-012-0604-1

Blewitt G (1990) An automatic editing algorithm for GPS data. Geophys Res Lett 17(3):199-202. doi:10.1029/GL017i003p00199
Bollerslev $\mathrm{T}$ (1986) Generalized autoregressive conditional heteroskedasticity. J Econom 31(3):307-327

Cai C, Liu Z, Xia P, Dai W (2013) Cycle slip detection and repair for undifferenced GPS observations under high ionospheric activity. GPS Solut 17(2):247-260. doi:10.1007/s10291-012-0275-7

Chen D, Ye S, Zhou W et al (2016) A double-differenced cycle slip detection and repair method for GNSS CORS network. GPS Solut 20(3):439-450. doi:10.1007/s10291-015-0452-6

CSNO (2013) BeiDou navigation satellite system signal in space interface control document, Open Service Signal (Version 2.0). China Satellite Navigation Office (CSNO), December 2013

de Lacy MC, Reguzzoni M, Sansò F, Venuti G (2008) The Bayesian detection of discontinuities in a polynomial regression and its application to the cycle-slip problem. J Geod 82:527-542. doi:10.1007/s00190-007-0203-8

de Lacy MC, Reguzzoni M, Sansò F (2012) Real-time cycle slip detection in triple-frequency GNSS. GPS Solut 16(3):353-362. doi:10.1007/s10291-011-0237-5

Goad C (1985) Precise positioning with the global position system. In: Proceedings of 3rd international symposium on inertial technology for surveying and geodesy, pp 745-756

Guo J, Xu X, Zhao Q, Liu J (2016) Precise orbit determination for quad-constellation satellites at Wuhan University: strategy, result validation, and comparison. J Geod 90(2):1-17. doi:10. 1007/s00190-015-0862-9

Hatch R (1982) The synergism of GPS code and carrier measurements. In: Proceedings of the third international symposium on satellite Doppler positioning at Physical Sciences Laboratory of New Mexico State University, 8-12 Feb, vol 2, pp 1213-1231

Huang L, Lu Z, Zhai G et al (2016) A new triple-frequency cycle slip detecting algorithm validated with BDS data. GPS Solut 20(4):761-769. doi:10.1007/s10291-015-0487-8

Ji S, Chen W, Weng D, Wang Z, Ding X (2013) A study on cycle slip detection and correction in case of ionospheric scintillation. Adv Space Res 51(2013):742-753. doi:10.1016/j.asr.2012.10.012

Kleusberg A, Georgiadou Y, van den Heuvel F, Heroux P (1993) GPS data preprocessing with DIPOP 3.0. Internal technical memorandum, Department of Surveying Engineering, University of New Brunswick, Fredericton, New Brunswick, Canada

Liu Z (2011) A new automated cycle slip detection and repair method for a single dual-frequency GPS receiver. J Geod 85(3):171-183. doi:10.1007/s00190-010-0426-y

Montenbruck O, Hauschild A, Steigenberger P, Hugentobler U, Teunissen P, Nakamura S (2013) Initial assessment of the COMPASS/BeiDou-2 regional navigation satellite system. GPS Solut 17(2):211-222. doi:10.1007/s10291-012-0272-x

Montenbruck O, Steigenberger P, Khachikyan R, Weber G, Langley RB, Mervart L, Hugentobler U (2014) IGS-MGEX: preparing the ground for multi-constellation GNSS science. Inside GNSS 9(1):42-49

Rousseeuw PJ, Croux C (1993) Alternatives to the median absolute deviation. J Am Stat Assoc 88(424):1273-1283. doi:10.1080/ 01621459.1993 .10476408

Wu Y, Jin S, Wang Z, Liu J (2010) Cycle slip detection using multifrequency GPS carrier phase observations: a simulation study. Adv Space Res 46(2):144-149. doi:10.1016/j.asr.2009.11.007

Xu PL (2005) Sign-constrained robust least squares, subjective breakdown point and the effect of weights of observations on robustness. J Geod 79:146-159. doi:10.1007/s00190-005-0454-1

Zhang X, Li P (2016) Benefits of the third frequency signal on cycle slip correction. GPS Solut 20(3):451-460. doi:10.1007/s10291015-0456-2 


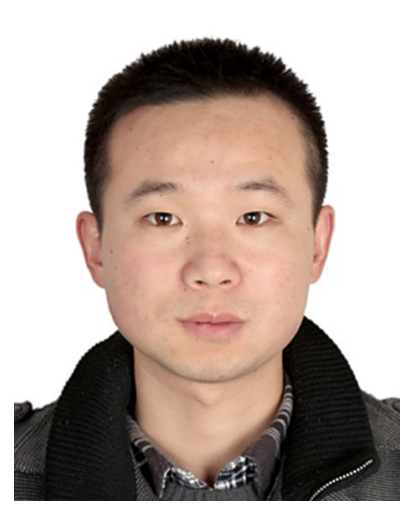

Bing Ju is a Ph.D. candidate in Applied Mathematics at the National University of Defense Technology in China. $\mathrm{He}$ received his B.S. and M.S. degrees from the National University of Defense Technology in 2009 and 2011, respectively. His current research activities are focus on multiGNSS data processing and precise orbit determination for low earth orbiters.
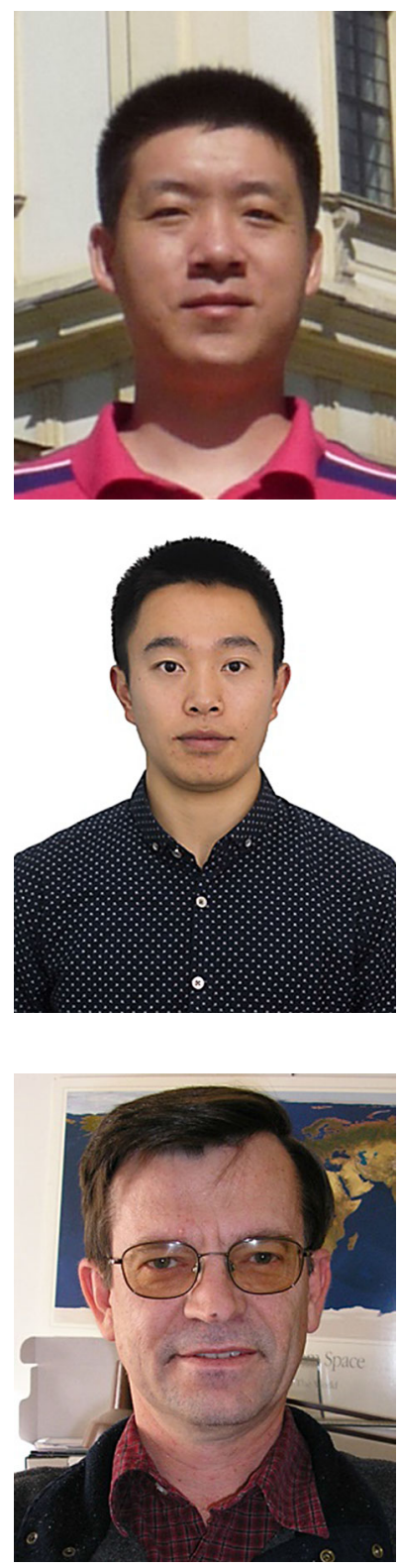

Thomas A. Herring received his Bachelor's and Master's degrees in geodetic science from the University of Queensland in Australia in 1976 and 1978. His Ph.D. in geophysics, awarded in 1983, is from MIT. Professor Herring joined the MIT faculty in 1989. His research interests are in the development and applications of precise geodetic techniques to study deformation processes on all spatial and temporal scales.

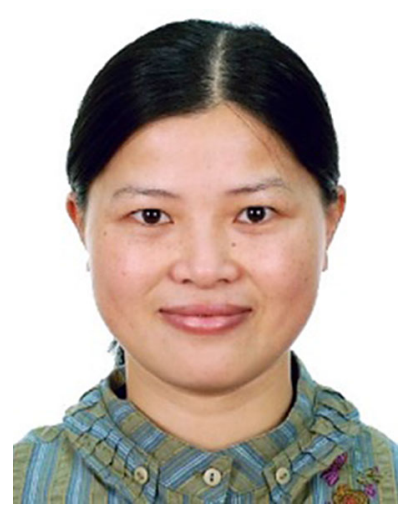

Xiaojun Duan is a professor at the National University of Defense Technology in China. She received her B.S. and M.S. degrees in Applied Mathematics and Ph.D. degree in System Engineering in 1997, 2000 and 2003, respectively. Her current research interests are in mathematical positioning, experimental design, and uncertainty quantification.

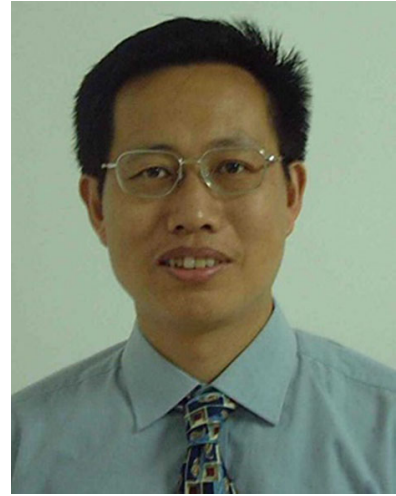

Zhengming Wang is a professor at the National University of Defense Technology in China. He received his B.S. and M.S. degrees in Applied Mathematics and $\mathrm{Ph} . \mathrm{D}$. degree in System Engineering in 1982, 1986 and 1998, respectively. His current research activities comprise mathematical modeling in tracking data, experimental design and evaluation, remote sensing image processing and data fusion theory. 